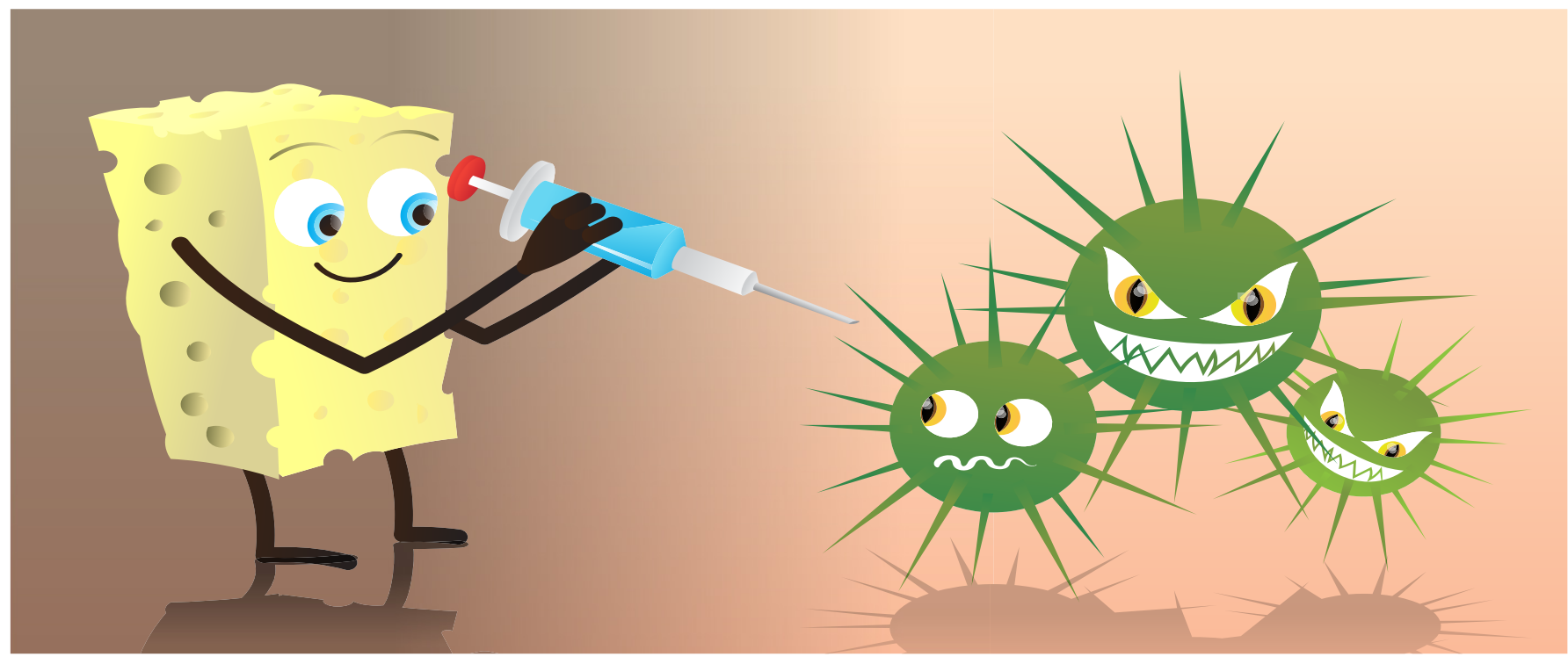

\title{
CAN WE USE NANOTECHNOLOGY TO
}

\section{TREAT CANCER?}

\section{Courtney R. Thomas*}

Faculté des Sciences de la Vie, École Polytechnique Fédérale de Lausanne, Lausanne, Switzerland

REVIEWED BY:

AIDAN

12 YEARS OLD
Could the next big change in cancer treatment actually be tiny? There are many reasons that cancer is such a difficult disease to treat. Scientists can either try to come up with new and better cancer medicines or they can find better ways for cancer patients to receive their medicines. This paper describes a possible new way to deliver cancer medicines using something called nanoparticles - tiny, tiny, sponge-like materials that have the cancer medicines inside. Scientists hope that nanoparticles will improve the delivery of the cancer medicine into tumors. By putting the medicine inside the nanoparticle, we can protect the healthy cells in the body from these strong medicines, and we might be able to use a lower dose of the medicine to treat the patient. This exciting technology is still being researched and improved, but could 1 day be used as an effective strategy to treat cancer patients.

\section{INTRODUCTION}

Although there have been many improvements in cancer treatments, cancer is still quite difficult to treat. One reason is because cancer medicine, called chemotherapy, can be quite difficult for a patient to receive. The medicines 
are quite strong, and patients taking chemotherapy can experience side effects such as tiredness, hair loss, weight loss, and pain. What if we were able to use a special device so that a patient with cancer could get the chemotherapy only in the cancer cells, without harming his or her healthy cells? This is the goal of researchers who are developing new ways to get drugs only to the cancer cells. These methods of getting the drugs to the cancer cells are called drug delivery vehicles (DDVs). The hope is that if chemotherapy can reach only the cancer cells and leave healthy cells undisturbed, maybe cancer patients could receive a lower dose of medicine, and have fewer of the strong side effects that are usually experienced with chemotherapy. Some kinds of DDVs are already being used to treat cancer patients, and some are still being developed in the research lab to improve them and make sure that they are safe before they can be tested in humans.

A major problem facing scientists who are trying to develop good DDVs is figuring out how to direct them into just the cancer cells. One solution is by taking advantages of some properties of cancer cells that are different from healthy cells [1].

\section{THE CANCER CELL}

Cancer is a complicated disease that causes cells to grow in a way that is different from normal, healthy cells. A normal cell in your body will divide until it becomes "old" or is damaged, when it dies in a special way called programed cell death. This dividing followed by programed cell death is a fine-tuned process that occurs in cells all over our bodies! But, cancer cells do not receive the signal to stop dividing, and as a result they have uncontrolled cell growth and can become a mass of cancer cells, called a tumor. Cells in a tumor have a survival advantage because tumors are often able to grow a lot of blood vessels, allowing them to get nutrients and continue growing. Another way that cancer cells differ from normal cells is that, because they are growing so rapidly, they can have more molecules on their surface, called surface receptors, than normal cells have. These receptors can recognize specific nutrients outside the cell and take them inside the cell to use to continue growing.

Researchers can take advantage of these differences between tumors/cancer cells and normal cells to try to direct a DDV into cancer cells and avoid healthy cells. DDVs are able to get inside tumors more easily than they can get into healthy tissues because the extra blood vessels make the tumors more permeable to the tiny DDV, and once the DDV gets inside the tumor it will stay there longer (Figure 1). This means that if a DDV can safely keep a cancer medicine inside of itself until it arrives at the tumor, this could be the key to a targeted cancer therapy, where the medicine is delivered into the cancer cells and not into healthy cells. 


\section{FIGURE}

Images $\mathbf{A}, \mathbf{C}$. show the nanoparticles using a special microscope called a transmission electron microscope (TEM) that allows us to see very tiny objects.

The nanoparticles in $\mathbf{A}$. are larger than the nanoparticles in C. Both nanoparticles have a molecule inside that makes them glow red under a special light. The larger nanoparticles $\mathbf{A}$. have a more difficult time getting inside of the tumor B. However, particles that are smaller $\mathbf{C}$. are able to reach the tumor and stay inside $\mathbf{D}$. We know that there are more of the smaller nanoparticles inside tumor compared with the larger nanoparticles because the tumor in $\mathbf{D}$. has a very strong red glow in the tumor.

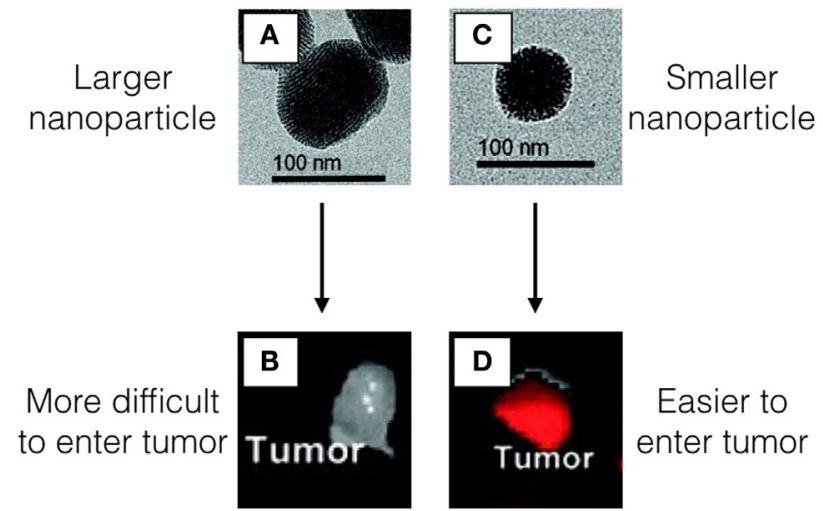

FIGURE 1

\section{DRUG DELIVERY VEHICLES MADE FROM NANOPARTICLES}

Based on what we know from the properties of cancer cells and tumors, there are several requirements for developing a DDV. First, the DDV must be made out of a material that should not be harmful to the person receiving it. Second, the DDV should be small enough to get into the tumor by way of the surface receptors and the blood vessels. Third, the DDV should be able to carry the cancer medicine inside of itself and keep it inside until it reaches the cancer cell, where the medicine needs to be released. A fourth requirement, which is not necessary for the patient but is necessary for scientists who want to study the DDV, is that we should be able to track the DDV in some way, so that we know where it goes in the body and whether it is able to get into the cancer cells.

In these experiments, researchers have used a type of DDV called nanoparticles. Nanoparticles are very tiny particles (about 1,000 times smaller than the diameter of one strand of your hair!). The nanoparticles used in this study are made out of silica, a type of glass, and they have small pores or holes, similar to a sponge, so that the cancer medicine can be stored inside.

We know that the smaller the nanoparticle is, the more easily it can stay inside the tumor [2]. This can allow the tumor to get a high dose of the cancer medicine when that medicine is released from the nanoparticle. Since the nanoparticles are like small sponges, we can easily get the medicines inside of them, but if the medicine does not stay in the nanoparticle until the nanoparticle reaches the tumor, then as soon as a patient gets the dose of nanoparticles, the medicine would slowly start to leak out! This will not protect the healthy cells or help us to get a high dose of medicine directly to the tumor.

A strategy we can use to keep the medicine inside of the nanoparticles until they get to the tumor is to connect some molecules to the surface of the nanoparticles to block the little openings in the "sponge" - these molecules 


\section{FIGURE 2}

The TEM allows us to see what the nanoparticles look like $\mathbf{A}$.

The nanoparticles have holes in them (that is how we can put the cancer medicines inside!), and the dark spots in the middle of the nanoparticle are the tiny pieces of metal that we put inside. The way that the nanoparticles work, shown in $\mathbf{B}$., is that we fill them with medicine (the red dots) and then block the holes with the gatekeeper molecules (the orange barrels). Then, when the nanoparticles get into the cancer cells, we can use heat to make the gatekeeper molecules move away from the nanoparticles, so that the small holes are unblocked and the cancer medicines can leak out of the nanoparticles and into the cancer cell. are called gatekeeper molecules. If we design the gatekeeper molecules, so that the openings in the nanoparticles are completely blocked, the medicine cannot leak out. We can create gatekeeper molecules that are able to change their shape in different conditions, so that when the nanoparticles arrive in the tumor, the gatekeeper molecules "open" the pores and the medicine can be delivered. If the gatekeeper molecules are not able to completely block the openings, or if they are able to change their shape before the nanoparticles arrive in the tumor, then some of the medicine will leak out, so it is important to have a well-designed gatekeeper.

One of the ways that we can change the shape of these gatekeeper molecules is by heating them. For this experiment, we use a special machine called an inductive heating machine. This machine has a special coil with an electric current inside, and the current creates a magnetic field around the coil. If you put a metal object like a screwdriver inside the coil, it can heat the metal to a really high temperature.

Fortunately, we do not need to heat the nanoparticles very much in order to get the gatekeeper molecules to change their shape! So, if we put a tiny piece of metal inside the nanoparticle, we can use this inductive heating machine to gently heat the area of the tumor, changing the shape of the gatekeeper molecules, so that the pores open and the medicine is delivered, but not heating it so much heat that it burns or kills cells (Figure 2).

Finally, we can make our nanoparticles fluorescent (they glow green when we use a special light to look at them), so that we can use a microscope to see where they are. This allows us to track them to see if they are able to go inside of the tumor.

\section{THE EXPERIMENT}

Once we were able to make complete nanoparticles with all of these qualities (cancer medicine inside, gatekeeper molecules on the surface, and tiny metal particles inside, so that we can use the inductive heating machine to release the medicine), we used them to test what happens inside of cancer cells. For this experiment, we did not use an animal or human with cancer, but instead tested

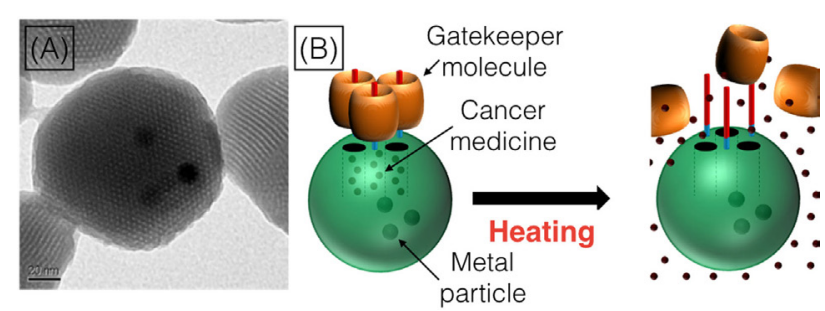

FIGURE 2 
FIGURE 3

In this experiment, we used breast cancer cells to see what would happen if we put our nanoparticles into cancer cells.

First, we wanted to see how much of the cancer medicine (a chemotherapy called doxorubicin, which looks red under the microscope) would be able to leak out from the nanoparticles. So, we added our nanoparticles to the cells and waited for 24 h. In A., you can see the nanoparticles as green spots, because we added a dye to the nanoparticles that allowed us to see where they go inside the cells, and the red is from the cancer medicine. As you can see in B., we saw that about $5 \%$ of the cells die under these conditions. Next, we wanted to see if the heat that we generate using our inductive heater is enough to kill the cells. In C., there is no red because we did not include the cancer medicine, but the green spots inside the cells tell us that the nanoparticles were able to go inside the cells. In 5 min, there was enough heat to kill about $17 \%$ of the breast cancer cells $\mathbf{D}$. even though these nanoparticles did not have any cancer medicines inside. This is because the heat produced by the tiny metal was enough to kill the cells. In the final experiment E., we can see that the nanoparticles are able to enter into the cells (the green spots inside the cells), and that the cancer medicine is able to leave the nanoparticles and enter the cell (the red color inside the cells). the nanoparticles on breast cancer cells that we could grow in the laboratory. We tried three different conditions with the breast cancer cells. We had one group of cells that got nanoparticles with medicine but did not get inductive heating, one that got nanoparticles with no medicine in them but they did get inductive heating, one that and one group that got nanoparticles AND inductive heating [3]. In Figure 3, you can see the outline of the breast cancer cells in gray. The yellow-green spots are our nanoparticles, and the red is the cancer medicine that has come out of the nanoparticles and entered into the cells. We hoped that when we used the complete nanoparticles as a DDV we would see the cancer cells dying!

When we gave the nanoparticles to the cells but did not turn on the heating instrument, we saw that about $5 \%$ of the breast cancer cells died (look at the left side of Figure 3). This means that some of the cancer medicine was leaking out of the nanoparticles. In the middle of Figure 3, we gave the cells nanoparticles with no cancer medicine inside, only the tiny metal pieces. When we put these cells inside the heating machine for $5 \mathrm{~min}$, about $17 \%$ of the cells died. This means that, even without medicine, the nanoparticles were generating enough heat to kill some of the breast cancer cells. Finally, when we combined both the cancer medicine and the metal pieces and heated the cells, we saw that $37 \%$ of the cancer cells died. We could see the cancer medicine inside the cells (in red), and we could also see that the cells became round, which is a sign of cell death.

\section{CONCLUSION}

The experiments told us that our nanoparticle DDV is a promising start for delivering cancer medicines into cancer cells, but there are ways that we should try to improve this system. We can re-design the nanoparticles to make
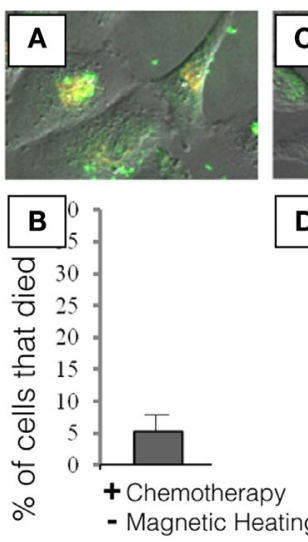
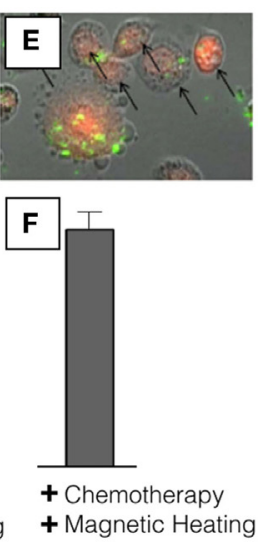
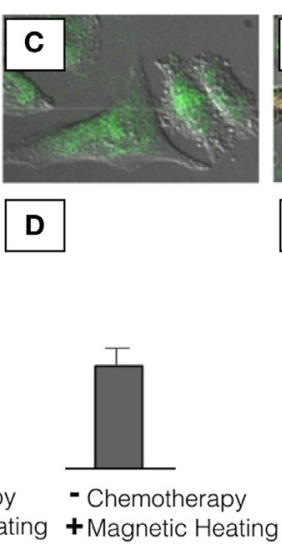

D 
FIGURE 3

\section{(CONTINUED)}

We also see that the shape of the cancer cells has changed a lot. Instead of being long like in $\mathbf{A}, \mathbf{C}$. the shape of the cell has changed to become round, indicating that the cell is dying. In this final experiment, we used nanoparticles that had the cancer medicine inside and we turned on our inductive heater for $5 \mathrm{~min}$ and we see that in this case, about $37 \%$ of the cancer cells died F. Since we had $5 \%$ of cells dying from cancer medicine leaking out without heating, and $17 \%$ of cells dying from the heating $(5+17=22 \%)$ we can see that, since $37 \%$ of cells died with the complete DDV, our method was able to allow some of the cancer medicine to be released into the cancer cells when they were heated, killing them from the inside. sure that none of the cancer medicine is leaking out before the nanoparticles reach the cancer cells. We can also try to decrease the amount of time that we need to turn on the heating machine to find a safe zone where none of the cells die from the heat. While there are some other nanoparticle DDVs already available for use in actual cancer patients, our method still needs some work before it can be tested in human patients. If our nanoparticle DDV can be improved enough to become an option for treating cancer patients, we hope that these patients will experience fewer of the negative side effects of chemotherapy than they have when the chemotherapy is given in the usual way. Maybe the next big change in cancer treatment can be a small, or nanosized, solution!

\section{REFERENCES}

1. Hanahan, D., and Weinberg, R. A. 2000. The hallmarks of cancer. Cell 100(1): 57-70. doi:10.1016/S0092-8674(00)81683-9

2. Meng, H., Xue, M., Xia, T., Ji, X., Tarn, D. Y., Zink, J. I., et al. 2011. Use of size and a copolymer design feature to improve the biodistribution and the enhanced permeability and retention effect of doxorubicin-loaded mesoporous silica nanoparticles in a murine xenograft tumor model. ACS Nano 5(5):4131-44. doi:10.1021/nn200809t

3. Thomas, C. R., Ferris, D. P., Lee, J. H., Choi, E., Cho, M. H., Kim, E. S., et al. 2010. Non-invasive remote-controlled release of drug molecules in vitro using magnetic actuation of mechanized nanoparticles. J. Am. Chem. Soc. 132(31):10623-5. doi:10.1021/ja1022267

SUBMITTED: 06 January 2016; ACCEPTED: 02 August 2016; PUBLISHED ONLINE: 17 August 2016.

EDITED BY: Fulvio D'Acquisto, Queen Mary University of London, UK

CITATION: Thomas CR (2016) Can We Use Nanotechnology to Treat Cancer? Front. Young Minds 4:12. doi:10.3389/frym.2016.00012

CONFLICT OF INTEREST STATEMENT: The author declares that the research was conducted in the absence of any commercial or financial relationships that could be construed as a potential conflict of interest.

COPYRIGHT @ 2016 Thomas. This is an open-access article distributed under the terms of the Creative Commons Attribution License (CC BY). The use, distribution and reproduction in other forums is permitted, provided the original author(s) or licensor are credited and that the original publication in this journal is cited, in accordance with accepted academic practice. No use, distribution or reproduction is permitted which does not comply with these terms. 


\section{REVIEWED BY}

\section{AIDAN, 12 YEARS OLD}

$\mathrm{Hi}$ I I am Aidan, and I live in San Francisco. I enjoy all types of life and physical science, sports, and taking care of toddlers. At school, I like science, history, and Chinese. I have gone to 16 countries on 6 cruises where I have learned a lot about their history, art, and science. I right now enjoy fencing most of my weekday afternoons.

\section{AUTHOR}

\section{COURTNEY R. THOMAS}

Courtney Thomas is a researcher at Ecole Polytechnique Fédérale de Lausanne (EPFL) on the shores of Lac Léman in Lausanne, Switzerland. She is currently researching how cancer grows and metastasizes in the body, and previously worked to develop new nanoparticles to better deliver medicines for cancer and tuberculosis. She is interested in learning how about cancer grows and new technologies for improved cancer therapy. ${ }^{*}$ courtney.thomas@epfl.ch 